\title{
DESARROLLO DEL RAZONAMIENTO \\ DEDUCTIVO A TRAVÉS DE LA \\ GEOMETRÍA EUCLIDIANA
}

\author{
Leonor Camargo Uribe \\ Carmen Samper de Caicedo*
}

\begin{abstract}
An approximation to the framework of the investigation project «Development of deductive reasoning through Euclidean Geometry» is presented. The project is in an initial stage and the ultimate goal is to validate a didactical model for the learning of Euclidean Geometry which emphasizes the development of deductive reasoning.
\end{abstract}

\section{INTRODUCCIÓN}

La identificación de la Educación Matemática y la Didáctica de la Matemática como campos académicos e investigativos desde un enfoque sistémico (escuela francesa), supera la concepción instrumental de didáctica, para situarla como campo investigativo, uno de cuyos frentes de trabajo es la producción teórica en matemáticas escolares y su validación mediante propuestas curriculares concretas.

Uno de los propósitos de la investigación que estamos iniciando es contribuir a la búsqueda de soluciones, desde la didáctica de la matemática, a los problemas de la enseñanza y aprendizaje de las matemáticas en la educación básica secundaria. Una de las metas es poder incidir en el diseño y desarrollo del currículo, en términos de buscar alternativas a la secuenciación y compartimentalización de los saberes matemáticos en la escuela, a través del reconocimiento de dominios conceptuales que se desarrollan en largos periodos de tiempo y a través de procesos de adquisición significativos.

Debido a la introducción de la "matemática moderna" en el currículo de la matemática escolar, en las décadas de los 70 y 80 en Colombia, la geometría, área de vital importancia en la educación matemática, sufrió un duro golpe en las reformas escolares al quedar relegada a un segundo plano, reducida en los libros de texto de matemáticas a una sola unidad y considerada como área de segunda categoría a la cual se le dedica un mínimo de tiempo; su potencial para desarrollar el pensamiento espacial o como herramienta para modelar situaciones problemáticas fue por varias décadas casi completamente ignorado. Por estas razones, nuestro centro de interés es la geometría euclidiana.

Con la nueva propuesta curricular en matemáticas planteada en los lineamientos curriculares del Ministerio de Educación Nacional (1998), que señalan como aspecto central de una posible estructura curricular para el trabajo en matemáticas, la búsqueda de un equilibrio entre los procesos que tienen que ver con el aprendizaje tales como el

\footnotetext{
*Profesoras Departamento de Matemáticas, Universidad Pedagógica Nacional.
} 
razonamiento y la resolución de problemas y los conocimientos básicos referentes a los procesos específicos que desarrollan el pensamiento matemático, la geometría y en particular el desarrollo del pensamiento lógico deductivo, se convierte en sustrato fundamental con el cual desarrollar la capacidad de reflexión, herramienta fundamental para explorar la realidad, representarla, explicarla, predecirla y actuar en ella Como marco de referencia, los lineamientos adoptan el punto de vista de David Perkins (1994) según el cual:

el objetivo de enseñarlas habilidades de pensamiento no se debería considerar como algo opuesto al de enseñar el contenido convencional sino como complemento de este. La capacidad de pensamiento y el conocimiento son como la trama y la urdimbre de la competencia intelectual, y el desarrollo de cualquiera de las dos cosas en detrimento de la otra, nos produciría algo muy distante de una tela de buena calidad" (p. 82).

Ante propuestas de esta naturaleza, corresponde a la investigación aportar a la búsqueda de alternativas curriculares que respondan a las nuevas exigencias sociales y a los planteamientos que la comunidad internacional ha ido adoptando frente al papel de la Educación Matemática.

El objetivo de esta presentación es dar a conocer una primera aproximación al marco teórico del proyecto de investigación: Desarrollo del razonamiento deductivo a través de la geometría euclidiana" que actualmente se encuentra en una etapa inicial y cuya meta apunta a la validación de un modelo didáctico para el aprendizaje de la geometría euclidiana, con énfasis en el desarrollo del pensamiento deductivo. Pensamos, con esta investigación, lograr un aporte a problemas de la enseñanza y el aprendizaje de la geometría en la Educación Básica secundaria. El esquema que se propone apunta al desarrollo de estrategias de razonamiento, a partir de actividades de construcción del conocimiento geométrico y a la integración de variables relativas a la cultura como a negociación de significados, contenidos flexibles, establecimiento de relaciones democráticas en torno al aprendizaje y preocupación por la comprensión acerca del conocimiento informal que los estudiantes llevan a la escuela.

Lo dicho anteriormente permite reconocer la contribución que la educación matemática debe realizar en la formación de ciudadanos, proporcionándoles herramientas matemáticas básicas para el desempeño social y profesional. Se integra así una difusión de valores democráticos y la realización del ejercicio de la crítica, como parte integrante de la cultura matemática escolar.

\section{MARCO TEÓRICO}

La estrategia para abordar la aproximación a la geometría euclidiana, como elemento básico en la formación del pensamiento deductivo, parte de una re-conceptualización sobre la disciplina matemática misma. En los procesos de construcción de matemáticas escolares, la matemática debe asumirse desde una perspectiva cultural, como una disciplina resultado de la actividad de grupos humanos ubicados en una sociedad, en un periodo histórico y en permanente cambio. La aproximación al sistema formal axiomático deductivo ha de partir de los conocimientos intuitivos para llegar a la formalización. El principio que sustenta la propuesta es que, por medio de experiencias manuales significativas, se hacen "descubrimientos" que llevan al establecimiento de conjeturas, las cuales posteriormente se demuestran. Sin desconocer que la esencia de la enseñanza de 
la geometría es llegar a la comprensión de un sistema deductivo, esta propuesta considera que éste debe ser el punto de llegada y no el punto de partida. De esta manera, el aprendizaje de la geometría se concibe como una actividad y no como un conjunto de contenidos a retener, pues existe la convicción según a cual, la comprensión no se alcanza con la información dada sino a través de la experiencia. Elemento importante a incorporar es la herramienta tecnológica, como un nuevo sistema de representación, donde los objetos matemáticos se "materializan" en elementos susceptibles de ser manipulados y transformados, dando así a la matemática un carácter casi "empírico".

Desde esta perspectiva, cualquier modelo didáctico que se proponga ha de basarse en investigaciones previas acerca del razonamiento geométrico y en investigaciones didácticas acerca de la construcción de conceptos geométricos en la escuela. En los apartes siguientes haremos mención de estos aspectos.

\section{El razonamiento en geometría}

El apoyo fundamental, para identificar elementos particulares del razonamiento, se derivará del trabajo realizado por los esposos Van Hiele (1957).

Los esposos, Dina van Hiele y Pierre Marie van Hiele, profesores de matemáticas en colegios de secundaria en Holanda, preocupados por lograr en sus alumnos comprensión de los conceptos en lugar de simple memorización de definiciones, demostraciones o algoritmos, realizaron un trabajo de investigación acerca de los procesos de aprendizaje en Geometría, el cual concluyó con sus respectivas tesis doctorales en 1957, en las cuales propusieron su modelo. El propio Pierre M. Van Hiele explicó así el origen de su interés por el tema (1986):

Cuando empecé mi carrera como profesor de Matemáticas, pronto me di cuenta de que era una profesión difícil. Había partes de la materia en cuestión que yo podía explicar y explicar y aún así los alumnos no entendían. Podía ver que ellos lo intentaban realmente, pero no tenían éxito. Especialmente al comienzo de la Geometría, cuando había que demostrar cosas muy simples, podía ver que ellos daban el máximo de sí, pero la materia parecía ser demasiado difícil. De pronto, parecía que comprendían la materia en cuestión. Podían hablar de ella con bastante sentido y a menudo decían:

"No es tan difícil, pero, ¿por qué nos lo explicó usted de forma tan complicada? En los años que siguieron cambié mi explicación muchas veces, pero las dificultades se mantenían. Parecía como si siempre estuviera hablando en una lengua distinta. $Y$ considerando esta idea descubrí la solución, los diferentes niveles de pensamiento."

La propuesta de los esposos Van Hiele está sustentada a partir de las siguientes afirmaciones:

- Es posible encontrar diferentes niveles de perfección en el razonamiento de los estudiantes en geometría (y en general en matemáticas).

- Un estudiante sólo podrá comprender realmente aquellas partes de las matemáticas que el profesor le presente de manera adecuada a su nivel de razonamiento.

- Si una relación matemática no puede ser expresada de forma comprensible, para el nivel de razonamiento actual de los estudiantes, es necesario esperar a que éstos alcancen un nivel de razonamiento superior para presentársela. 
- No se puede enseñar a una persona a razonar de una determinada forma sólo se aprende a razonar mediante la propia experiencia. En lugar de ello, se puede ayudar a esa persona, a adquirir, lo antes posible, la experiencia necesaria para que llegue a razonar de esa manera.

Así pues, los van Hiele enfatizan en la existencia de diferentes formas de razonamiento y señalan la necesidad de que los profesores tengan en consideración la capacidad de razonamiento de sus alumnos al decidir la forma y el rigor de sus clases.

El cuadro número 1 recoge las características principales de los cuatro primeros niveles de razonamiento matemático de Van Hiele e indicadores del logro en los estudiantes. El nivel 5 (Rigor) no se presenta pues supone un tratamiento geométrico que supera las ambiciones de la Educación Básica.

\section{Nivel 1 Reconocimiento}

\begin{tabular}{|c|c|}
\hline Características & Indicadores \\
\hline $\begin{array}{l}\text { Percepción global de las figuras, en su } \\
\text { totalidad, } \\
\text { Inclusión de atributos irrelevantes en las } \\
\text { descripciones, generalmente de tipo físico o } \\
\text { visual (orientación, tamaño, etc). } \\
\text { Referencia a prototipos visuales para } \\
\text { caracterizar las figuras. } \\
\text { Percepción individual de cada figura. No se } \\
\text { generalizan las características a otras figuras de } \\
\text { la misma clase. } \\
\text { No se reconocen explícitamente las partes de } \\
\text { que se componen las figuras ni sus propiedades } \\
\text { matemáticas. } \\
\text { No se utiliza lenguaje geométrico apropiado. }\end{array}$ & $\begin{array}{l}\text { Uso de cualidades imprecisas para } \\
\text { comparar, identificar, caracterizar y } \\
\text { escoger figuras: "está torcido", "está } \\
\text { deforme" "este lado es mas largo", "es } \\
\text { mas gordo que", etc. } \\
\text { Caracterización de figuras mediante ex- } \\
\text { presiones como: "parece un cono boca } \\
\text { abajo", "tiene forma de casita", etc. } \\
\text { Descripciones basadas en semejanzas } \\
\text { físicas globales: "Se parece a una } \\
\text { puerta "Tiene forma de cono".. } \\
\text { Realización de selecciones incorrectas } \\
\text { a partir de propiedades que no } \\
\text { pertenecen realmente a la figura: } \\
\text { "Todos los rombos tienen una punta } \\
\text { hacia arriba" } \\
\text { Uso incorrecto de las propiedades } \\
\text { como condiciones necesarias al } \\
\text { determinar una figura: Al adivinar con } \\
\text { pistas, utilizan sólo algunas de ellas y } \\
\text { olvidan las demás. }\end{array}$ \\
\hline
\end{tabular}

\section{Nivel 2 Clasificación}

\begin{tabular}{|c|c|}
\hline Características & Indicadores \\
\hline $\begin{array}{l}\text { Comienzos de razonamiento formal en } \\
\text { matemáticas. Reconocimiento de las } \\
\text { reaciones entre propiedades. } \\
\text { Reconocimiento de implicaciones: posibilidad } \\
\text { de clasificar lógicamente diferentes familias de } \\
\text { figuras a partir de sus propiedades y relaciones } \\
\text { ya conocidas. No obstante sus razonamientos }\end{array}$ & $\begin{array}{l}\text { Una reacción típica de los estudiantes } \\
\text { del segundo nivel o de los inferiores es } \\
\text { que, ante la petición del profesor de que } \\
\text { demuestren alguna propiedad, le } \\
\text { contesten: } \\
\text { “Por qué tenemos que demostrarla, } \\
\text { si ya sabemos que es verdad?” }\end{array}$ \\
\hline
\end{tabular}


lógicos, el apoyo es la manipulación.

Descripción de manera formal: definiciones matemáticas correctas, comprensión del papel de las definiciones y los requisitos de una definición correcta.

Comprensión de los sucesivos pasos individuales de un razonamiento lógico formal de manera aislada, ya que no se entiende la necesidad del encadenamiento de pasos ni la estructura de la demostración. Los alumnos pueden entender una demostración explicada por el profesor o desarrollada en un libro de texto pero no construirla por si mismos. Identificación de las propiedades necesarias y suficientes.
En las demostraciones, no se contentan con la comprobación de unos pocos casos. Hay una necesidad de dar alguna consistencia mayor en términos de presentación de una justificación general o de cubrir de alguna manera los posibles casos que se puedan presentar.

Forman definiciones completas de tipos y formas y son hábiles para modificar definiciones y aceptar usar definiciones equivalentes de los conceptos.

Hacen referencia explícita a las definiciones.

Aceptan los ordenamientos lógicos parciales entre tipos de figuras incluida la inclusión de clases.

Son hábiles para escoger formas, de acuerdo a una serie de atributos matemáticos precisos.

Usan, explícitamente, las expresiones condicionales: "si, entonces".

Forman argumentos deductivos informales, usando formas lógicas como la ley transitiva $[p \rightarrow q$ y $q \rightarrow r] \rightarrow(p \rightarrow r)$ y la ley de separación modus ponens $[(p \rightarrow$ $q \wedge p) \rightarrow q]$.

Confunden los roles de axiomas y teoremas. Al no ser capaces de realizar razonamientos lógicos formales por su propia cuenta, ni sentir su necesidad, los estudiantes no comprenden la estructura axiomática de las matemáticas.

\section{Nivel 3 Análisis}

\begin{tabular}{|c|c|}
\hline cas & IS \\
\hline $\begin{array}{l}\text { Razonamiento formal. } \\
\text { Comprensión y desarrollo de } \\
\text { demostraciones formales. } \\
\text { Comprensión de la estructura } \\
\text { axiomática de las matemáticas: } \\
\text { sentido de axiomas, teoremas, } \\
\text { términos definidos, términos no } \\
\text { definidos. } \\
\text { Aceptación de la posibilidad de llegar } \\
\text { al mismo resultado desde distintas } \\
\text { premisas. } \\
\text { Necesidad de la demostración como } \\
\text { único medio para verificar la verdad de } \\
\text { una afirmación, }\end{array}$ & $\begin{array}{l}\text { Exigen plantear preguntas ambiguas y tareas } \\
\text { problemáticas en lenguaje preciso. } \\
\text { Realizan frecuentes conjeturas e intentos de } \\
\text { verificarlas deductivamente. } \\
\text { Construyen demostraciones y ven la posibilidad } \\
\text { de desarrollar una demostración de distintas } \\
\text { maneras. Pueden comprender y contrastar } \\
\text { diferentes demostraciones de un mismo teorema. } \\
\text { Confían en la comprobación como la autoridad } \\
\text { final. } \\
\text { Diferencian el rol de los componentes de un } \\
\text { discurso matemático: axiomas, definiciones, } \\
\text { teoremas, } \\
\text { Identifican las interacciones entre las condiciones }\end{array}$ \\
\hline
\end{tabular}


Capacidad de razonamiento lógico necesarias y las suficientes y distinguen entre matemático y visualización globalizadora del área de geometría.

una implicación $(p \rightarrow q)$ y su recíproca $(q \rightarrow p)$. Aceptan implícitamente los postulados de la geometría euclidiana.

\section{Nivel 4. Deducción Formal}

\begin{tabular}{|c|c|}
\hline Características & Indicadores \\
\hline $\begin{array}{l}\text { Comparación de sistemas basados en } \\
\text { diferentes axiomas. } \\
\text { Máximo nivel de rigor matemático según } \\
\text { los parámetros actuales. }\end{array}$ & $\begin{array}{l}\text { Posibilidad de diferenciar varias geometrías en } \\
\text { ausencia de modelos concretos. }\end{array}$ \\
\hline
\end{tabular}

Cuadro 1

Uno de los presupuestos para el desarrollo de este trabajo, manifiesto en las dificultades detectadas en diferentes evaluaciones escolares sobre el desempeño en razonamiento deductivo, es la identificación de un vacío curricular en actividades de aprendizaje que apunten al desarrollo de competencias propias de los niveles 1 al 3 . Por esto, la propuesta enfatiza en el acercamiento geométrico apropiado a estos niveles.

\section{Propiedades de los niveles}

- Un nivel no es exclusivo de una cierta edad o curso. Cada vez que se presenta un nuevo concepto, los estudiantes pasan por cada nivel, si bien, algunas veces el paso es más o menos lento.

- Los niveles están jerarquizados por grados de sofisticación en el razonamiento matemático que puede usar una persona.

- Cada nivel de razonamiento se apoya en el anterior. Pensar en el segundo nivel no es posible sin tener la capacidad de razonamiento del primero.

- Los niveles tienen una estructura recursiva. Hay habilidades que se usan implícitamente por los estudiantes y cuyo uso explicito se logra en el siguiente nivel (ver tabla 1).

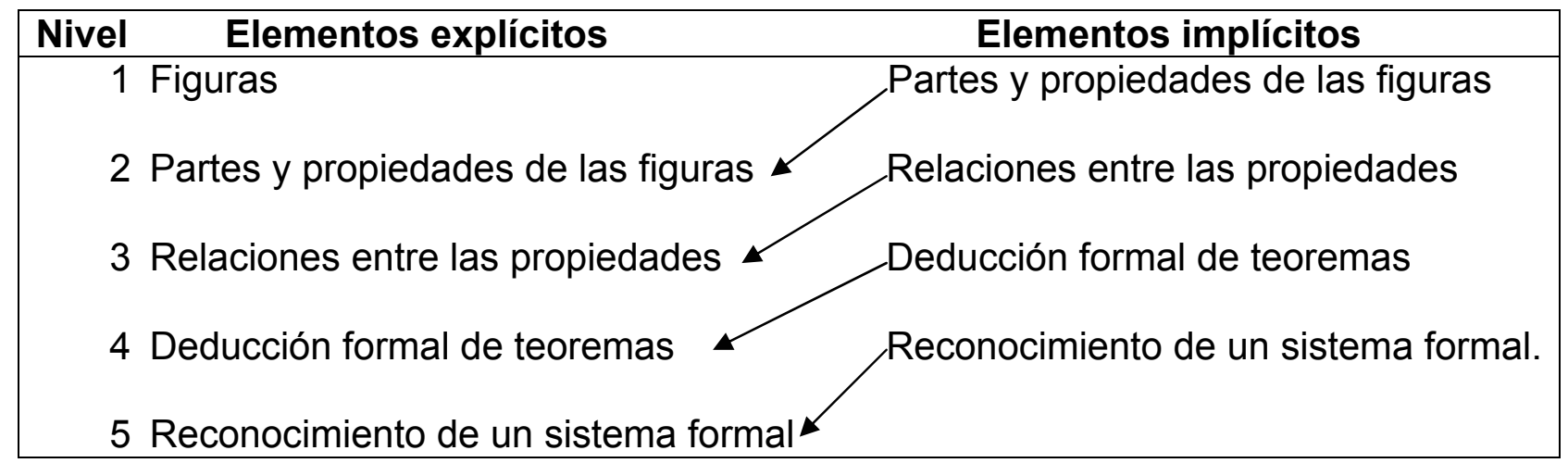


- Las actividades propuestas a los estudiantes deben estar orientadas a explicitar y tomar conciencia de los elementos implícitos.

- Hay una estrecha relación entre el lenguaje y los niveles. En cada uno hay diferentes formas de expresión y significado dado al vocabulario. Por ejemplo, en el nivel 2 los conjuntos de cuadrados y de rectángulos son disyuntos, mientras que en el nivel 3 el conjunto de los cuadrados está contenido en el de los rectángulos. El término comprobar significa, en el nivel 2, verificar, por ejemplo, por medio de mediciones que la afirmación es cierta en unos pocos casos, incluso en uno sólo, mientras que en el nivel 3 se realizan razonamientos lógicos, aunque con argumentos informales, basados en observación de ejemplos concretos. Esto se traduce a veces en demostraciones incorrectas (falacias geométricas). Sólo en el nivel 4, comprobar implica realizar un proceso deductivo con el rigor necesario.

- El paso de un nivel al siguiente se produce en forma continua.

\section{Propuestas didácticas}

Dentro de las investigaciones en didáctica que se utilizarán para configurar la propuesta didáctica se reseñan los trabajos de Hercowitz y Vinner (1987), Kalin y Corbett (1990) y Gutiérrez (1996). Todos ellos señalan, como elemento fundamental, el trabajo profundo para lograr la comprensión de las definiciones de conceptos geométricos. Utilizaremos la propuesta de Gutierrez para configurar actividades didácticas que lleven a la construcción de conceptos geométricos. Esta propuesta se presenta en el cuadro 2.

Gutiérrez basa su propuesta en los trabajos de Vinner acerca de la formación de conceptos y su relación con las definiciones matemáticas. Al trabajar un concepto geométrico, las personas se forman una idea de éste (imagen conceptual) que no siempre coincide con la que se deriva de su definición. Tener una idea "completa" de un concepto no significa tener en la mente unos pocos ejemplos sino que incluye las propiedades matemáticas de éste y sus relaciones con otros conceptos. Como cualquier ejemplo concreto siempre posee las características propias del concepto (propiedades relevantes) junto con otras que no son necesarias para caracterizarlo (propiedades irrelevantes), en la construcción de situaciones didácticas para la enseñanza de un concepto geométrico dado han de proponerse ejemplos que abarquen un gran rango de posibilidades para ir descartando las propiedades irrelevantes y configurando la noción completa del concepto trabajado. 


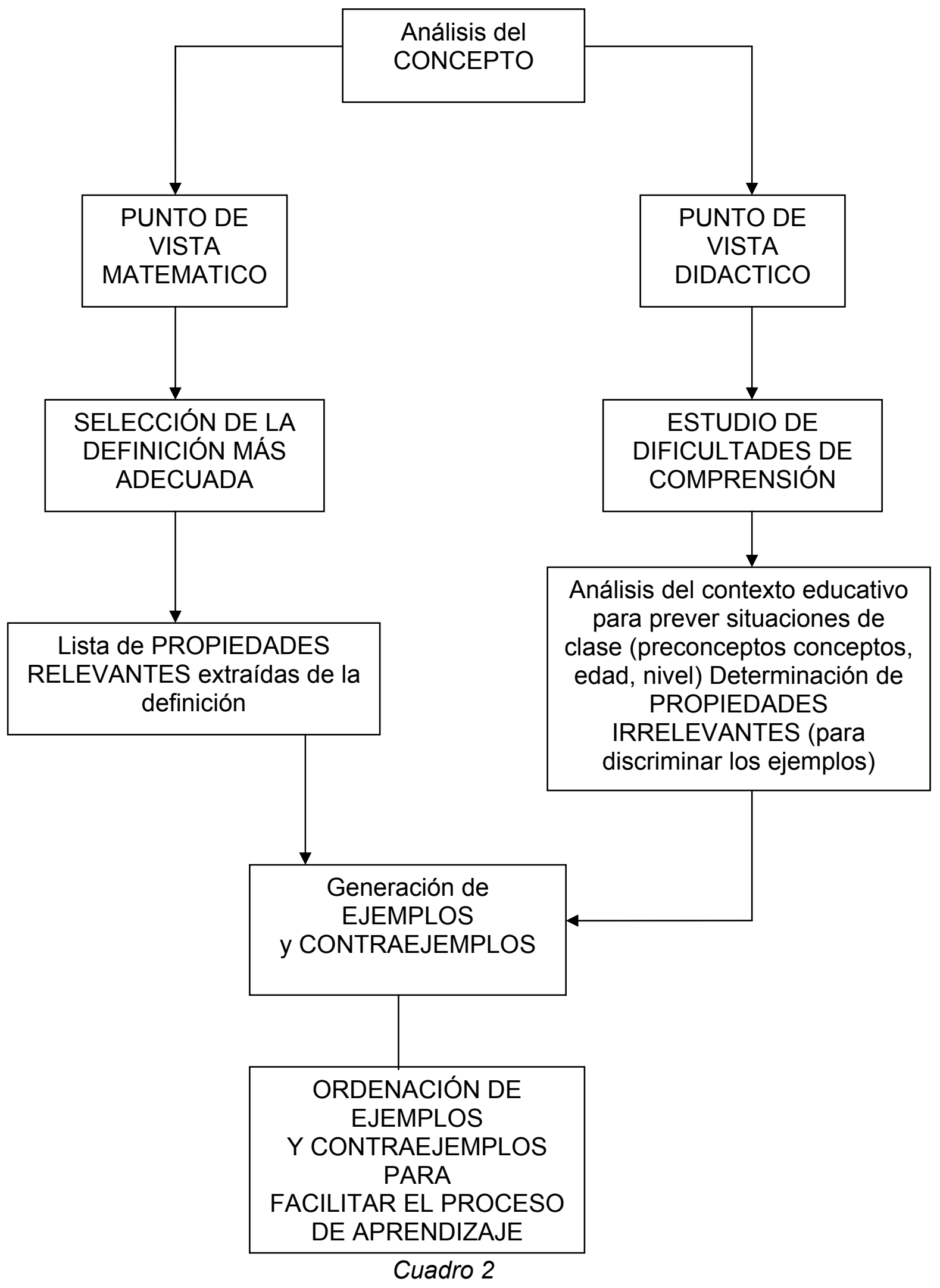

\section{FASES DEL PROYECTO}

En una primera fase, el proyecto se centrará en la determinación de competencias previas al razonamiento propiamente deductivo que sirvan de puente, como señalan los esposos Van Hiele, entre el pensamiento informal y el formal, enfocando la atención en 
aspectos específicos del estudio de polígonos. De acuerdo a cada competencia, se elaborarán actividades didácticas para detectar y desarrollar dichas competencias.

La segunda fase apuntará al desarrollo de competencias propias del razonamiento deductivo.

\section{ASPECTOS METODOLÓGICOS}

El método seleccionado para llevar a cabo la investigación corresponde a la categoría de métodos cualitativos. Las técnicas para registrar I información corresponderán a estudios de caso, entrevistas, observaciones directas, registro de lo sucedido, evidencias documentadas etc. Para validar el modelo, hasta el momento se tiene prevista la construcción, a partir del estudio de las competencias previas requeridas para superar cada una de las fases, de unidades de análisis para la organización de los contenidos (establecen los aspectos concretos de contenido y las situaciones problema que se proponen), unidades de análisis para la comprensión del contenido (permiten establecer los distintos aspectos cognitivos que se ponen en juego a los alumnos) y unidades de análisis para la interacción didáctica (permiten estudiar las interacciones que tienen lugar entre el proceso y los estudiantes a lo largo del proceso didáctico y su relación con la construcción).

La fiabilidad de la investigación se sustentará en la fiabilidad externa e interna características de la investigación cualitativa.

\section{BIBLIOGRAFIA}

CROWLEY, M. (1987). The Van Hiele Model of the development of geometric thought. En NCTM Learning and Teaching geometry K-12 (1987) Yearbook. Reston.

GUTIERREZ A., JAIME A. (1989). Bibliografía sobre el modelo de razonamiento geométrico de Van Hiele. Revista Enseñanza de las Ciencias, 7(1), 89-95

PERKINS, D.(1994). Enseñar a pensar, Ediciones Paidos

JAIME, A. GUTIÉRREZ, A. (1990). Una propuesta de fundamentación para la enseñanza de la geometría: El modelo de Van Hiele. En Linares 5, Sánchez, M.: Teoría y práctica en Educación Matemática. Ediciones Alfar, Sevilla.

JAIME, A. GUTIÉRREZ, A. (1998). Geometría y algunos aspectos generales de la Educación Matemática. Una empresa Docente. Universidad de los Andes.

SERRA, M. (1998) .Discovering Geometry: an inductive approach. Key Curriculum Press. San Francisco.

ACUÑA, C. (1996).Un modelo de tratamiento didáctico para la enseñanza de la geometría en el nivel medio superior En Espinosa F: Investigaciones en Matemática educativa. Grupo Editorial Iberoamérica. México. 
FARREL, M. (1987).Geometty for Secondaty School Teachers. En NCTM Learning and Teaching geometry K-12 (1987) Yearbook. Reston

HERSHKOWITZ, R., VINNER, 5., BRUCKEIMER, M. (1987).Activities with Teachers Based en cognitive research.. En NCTM Learning and Teaching geometry K-12 (1987) Yearbook. Resten. 